\title{
Rubber Wear and the Role of Transfer Films on Rubber Friction on Hard Rough Substrates
}

\author{
A. Tiwari ${ }^{1,3} \cdot$ N. Miyashita ${ }^{2} \cdot$ B. N. J. Persson ${ }^{1,3}$
}

Received: 18 December 2020 / Accepted: 5 February 2021 / Published online: 9 March 2021

(c) The Author(s) 2021

\begin{abstract}
We study the influence of rubber transfer films on the sliding friction between rectangular rubber blocks and a concrete surface. We present experimental results for the friction coefficient for a rubber compound sliding on a concrete surface contaminated by another rubber compound, for two different pairs (A, B) and (C, D) of rubber compounds. For the same rubber compounds, we present theory results which illustrate the relative importance of the viscoelastic and adhesive contribution to the sliding friction. We correlate the calculated rubber friction with the nature of the observed transfer films (or wear processes).
\end{abstract}

\section{Graphical Abstract}
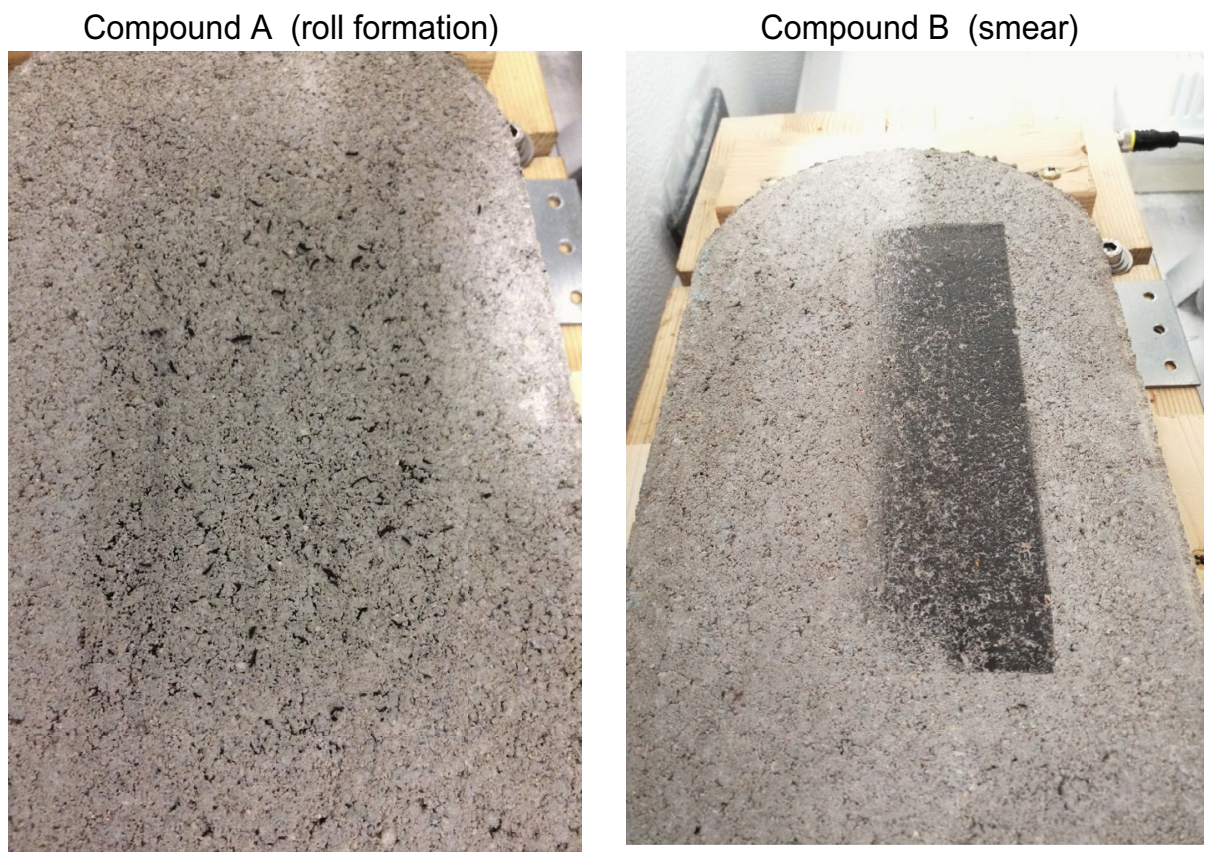

During sliding, compound $A$ wears by formation of a loosely bound rubber powder, while compound B forms a strongly bound smear film. We attribute the different wear modes to different frictional processes, where the friction is dominated by the viscoelastic contribution for compound $A$, and by the adhesive contribution for compound B.

Keywords Rubber wear · Contamination film · Racing tires - Adhesive wear--insert

Extended author information available on the last page of the article 


\section{Introduction}

It is often stated that in F1-racing transfer of rubber to the racing track can have a strong influence on the sliding friction or grip. In particular, it is claimed that when a race track is contaminated with a transfer film from one type of tire, changing to another type of tire may result in smaller friction than would be the case if the road surface would not have been contaminated by the first type of tire. In this study, we show that this statement is in general not true, at least not for dry road surfaces during steady sliding at low slip speed. We consider two pairs of rubber compounds (A, B) and $(C$, D) sliding on four different but nominally identical dry concrete surfaces. We show that the nature of the contamination film may be important when predicting if the friction for a second rubber compound will increase or decrease by the contamination film. Hence a smear film results in a reduction in the friction while when the wear occurs by formation of loose rubber particles the friction is nearly unchanged or increased. For the same four rubber compounds we present theory results which illustrate the relative importance of the viscoelastic and adhesive contribution to the friction. We interpret the adhesive contribution as arising from molecular stick-slip processes at the interface between a rubber transfer film and the rubber block, where most of the slip is assumed to occur. We observe different form of the transfer films (wear processes) for compound $\mathrm{A}$ and $\mathrm{B}$, which we attribute to differences in the relative importance of the viscoelastic and adhesive contribution to the friction.

\section{Experimental}

Using a linear friction tester (see Fig. 1) we have measured the sliding friction force when rectangular rubber blocks are sliding on concrete surfaces. The concrete surfaces have been prepared in a nominal identical way. The size of the rubber block is $A_{0}=3 \mathrm{~cm} \times 7 \mathrm{~cm}$ (and thickness $0.5 \mathrm{~cm}$ ), and the normal force $F_{0}=250 \mathrm{~N}$ giving the nominal contact

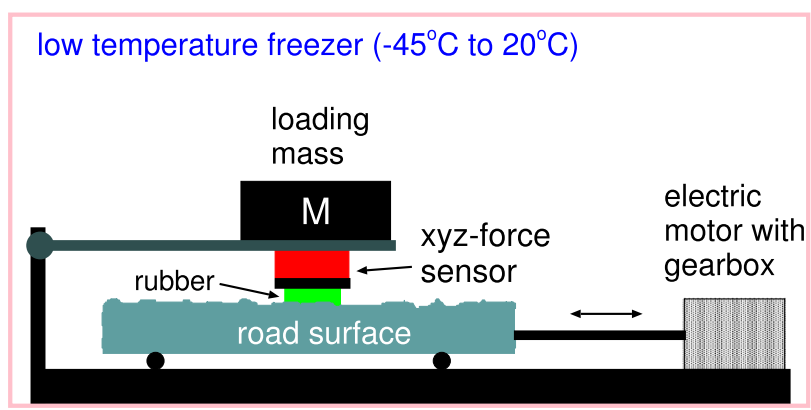

Fig. 1 Schematic picture of low-temperature friction instrument allowing for linear reciprocal motion pressure $p_{0}=F_{0} / A_{0} \approx 0.12 \mathrm{MPa}$. The friction tester can be operated at temperatures from room temperature down to $-45^{\circ} \mathrm{C}$, and the sliding speed from $1 \mu \mathrm{m} / \mathrm{s}$ (or less) to $1 \mathrm{~cm} / \mathrm{s}$. In the present study, all measurements were performed at $T=20^{\circ} \mathrm{C}$.

A rectangular rubber block is glued into the milling grove of the sample holder which gets attached to the force cell (red block in the figure). The rubber specimen can move with the carriage in the vertical direction to adapt to the substrate profile. The normal load $F_{0}$ can simply be changed by adding additional steel weights on top of the force cell. The substrate sample gets attached to the machine table which is moved by a servo drive via a gearbox in a translational manner. Here we control the relative velocity between the rubber specimen and the substrate sample while the force cell acquires information about normal force $F_{0}$ as well as friction force $F_{\mathrm{f}}$. Below we report the friction coefficient $\mu=F_{\mathrm{f}} / F_{0}$.

We have performed experiments using 4 different types of tread rubbers denoted A,B,C and D. Some information about the rubber compounds are given in Tables 1 and 2 and more details in Appendix A. The rubber elongation at break (strain $\left.\epsilon_{\mathrm{B}}=\Delta L / L_{0}\right)$ and tensile strength $\left(\sigma_{\mathrm{B}}\right)$ given in Table 2 may reflect the nucleation and propagation of cracks, but there is

Table 1 Summary of the glass transition temperatures of the tire tread compounds A-D

\begin{tabular}{lll}
\hline Compound & $T_{\mathrm{g}}$ in ${ }^{\circ} \mathrm{C}$ & $\begin{array}{l}\text { Maxi- } \\
\text { mum of } \\
\tan \delta\end{array}$ \\
\hline A (SBR I, racing, dry) & -12.9 & 0.68 \\
B (NR, truck, bus) & -65.5 & 0.84 \\
C (SBR I, racing, dry) & -33.5 & 0.78 \\
D (SBR II, racing, wet) & -24.4 & 0.97 \\
\hline
\end{tabular}

Compound A is a SBR I racing compound for dry surfaces with $19 \mathrm{vol} \%$ carbon black and $15 \mathrm{vol} \%$ oil. Compound B is a NR truck/bus compound with $20 \mathrm{vol} \%$ carbon black. Compound C is a SBR I racing compound for dry surfaces with 20vol\% carbon black and 32vol\% oil. Compound D is a SBR II racing compound for wet surfaces with $20 \mathrm{vol} \%$ silica and $29 \mathrm{vol} \%$ oil. The glass transition temperature is defined as the maximum of $\tan \delta$ as a function of temperature for the frequency $\omega_{0}=0.01 \mathrm{~s}^{-1}$

Table 2 Elongation at break (strain $\epsilon_{\mathrm{B}}=\Delta L / L_{0}$ ) and tensile strength $\left(\sigma_{\mathrm{B}}\right)$ of the tire tread compounds A-D at the strain rate $0.12 \mathrm{~s}^{-1}$

\begin{tabular}{lll}
\hline Compound & $\epsilon_{\mathrm{B}}$ & $\sigma_{\mathrm{B}}(\mathrm{MPa})$ \\
\hline A (SBR I, racing, dry) & 6.52 & 13.9 \\
B (NR, truck, bus) & 4.72 & 29.6 \\
C (SBR I, racing, dry) & 7.05 & 11.9 \\
D (SBR II, racing, wet) & 6.59 & 11.2 \\
\hline
\end{tabular}


no clear correlation between these quantities and the wear mode (and wear rate) discussed below.

As substrates, we have used 4 different but nominal identical concrete blocks. We have used these concrete surfaces in many earlier studies (see, e.g, [1-3]). The concrete blocks are produced in the same way and appear to the naked eye the same. We have measured the surface topography and calculated the power spectra of such surfaces in several earlier studies, and the power spectra are always the same within the noise of the measurements.

\section{Experimental Results}

We have performed a set of experiments to study the role of rubber contamination films on rubber friction on concrete surfaces. We consider two pairs of rubber compounds $(A, B)$ and $(C, D)$ sliding on four different but nominally identical concrete surfaces.

\subsection{Compound A and B}

In the experiments, we first run-in the rubber compounds A and $\mathrm{B}$ on two different concrete surfaces $\mathbf{a}$ and $\mathbf{b}$. The runin consisted of 15 forwards and backwards sliding events (sliding speed $v=1 \mathrm{~cm} / \mathrm{s}$ ), each $20 \mathrm{~cm}$ long, of compound A on surface $\mathbf{a}$, and similar for compound B on surface $\mathbf{b}$. Fig. 2 shows the friction coefficient as a function of the number of sliding cycles for compound A (red) and B (blue) sliding on the concrete surfaces $\mathbf{a}$ and $\mathbf{b}$. The two concrete surfaces are nominally identical so the small difference in the friction observed may be attributed to the different rubber compounds. Note that during run-in there is a decrease

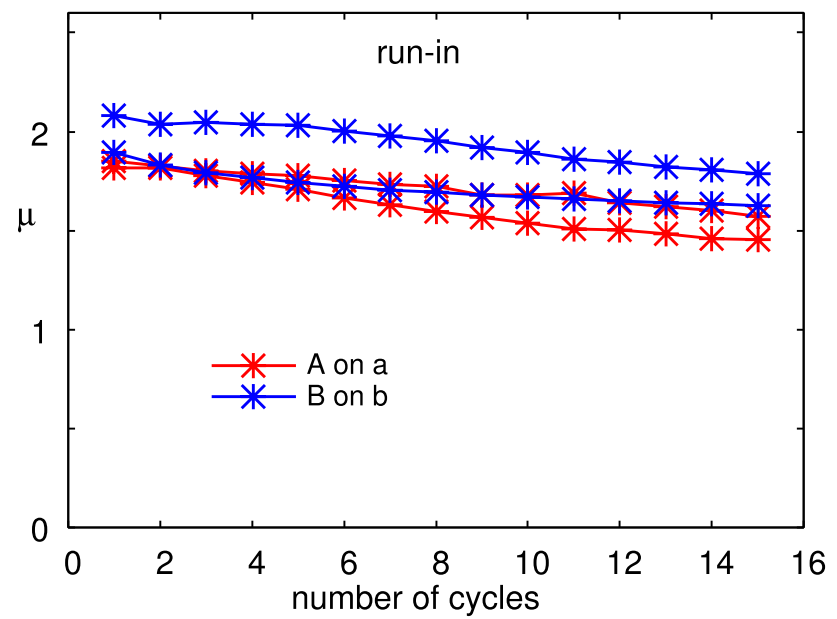

Fig. 2 The friction coefficient at the sliding speed $v=1 \mathrm{~cm} / \mathrm{s}$ as a function of the number of sliding cycles for compound A (red) and B (blue) sliding on two different concrete blocks denoted $\mathbf{a}$ and $\mathbf{b}$, respectively. In both cases, run-in was performed two times in the friction by $\sim 20 \%$ in both cases. This may be due to the influence on the friction by a thin contamination film deposited on the concrete surface, or the removal of a thin surface layer on the rubber block, or the increased roughness of the rubber surface.

Figure 3 shows a picture of (a) the concrete block a after run-in of compound A, and (b) the concrete block $\mathbf{b}$ after run-in of compound B. Note the difference in wear mode: compound A wear mainly by the formation of wear particles, which are easily removed with a soft brush or a vacuum cleaner, while compound $\mathrm{B}$ wear by formation of a thin smear film which adhere strongly to the concrete surface. As will be shown in Sec. 4 this is consistent with the (theoretically predicted) origin of the rubber friction which for $T=20^{\circ} \mathrm{C}$ and the sliding speed $v=1 \mathrm{~cm} / \mathrm{s}$, is mainly viscoelastic for compound $\mathrm{A}$ and adhesive for compound $\mathrm{B}$.

Next we measured the friction force for compound A on surface a for sliding speeds from $1 \mu \mathrm{m} / \mathrm{s}$ to $1 \mathrm{~cm} / \mathrm{s}$, and the same experiment for compound B on the surface $\mathbf{b}$. The results are given by the solid red and blue lines in Fig. 4 . Next we measured the friction for the same velocity interval for compound $\mathrm{A}$ on the concrete surface $\mathbf{b}$ contaminated by compound B (red dashed line in Fig. 4). Finally, we performed a similar experiment for compound $\mathrm{B}$ on the concrete surface a already contaminated by the compound A (blue dashed line in Fig. 4). As shown in the figure the friction coefficient for compound A, when sliding on surface $\mathbf{a}$ contaminated by compound $\mathrm{A}$, is slightly larger than when sliding on surface $\mathbf{b}$ contaminated by the compound B (red lines). The opposite is true for compound B (blue lines). These results indicate that the nature of the contamination film may be important when predicting if the friction for a second rubber compound will increase or decrease by the contamination film. Hence a smear film results in a reduction in the friction while when the wear occurs by formation

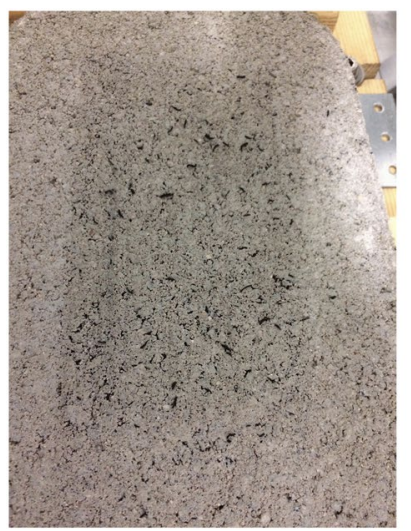

(a) run-in $A$ on a (roll formation) $[\mathrm{V}=1 \mathrm{~cm} / \mathrm{s}, 15$ cycles]

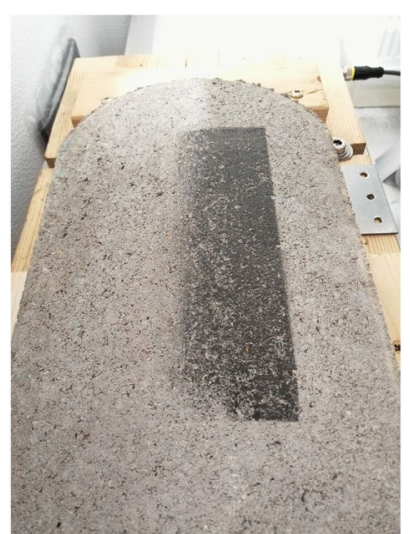

(b) run-in B on b (smear) $[\mathrm{v}=1 \mathrm{~cm} / \mathrm{s}, 15$ cycles $]$
Fig. 3 Pictures of concrete blocks after run-in of (a) compound A on concrete block $\mathbf{a}$ and (b) compound B on concrete block b 


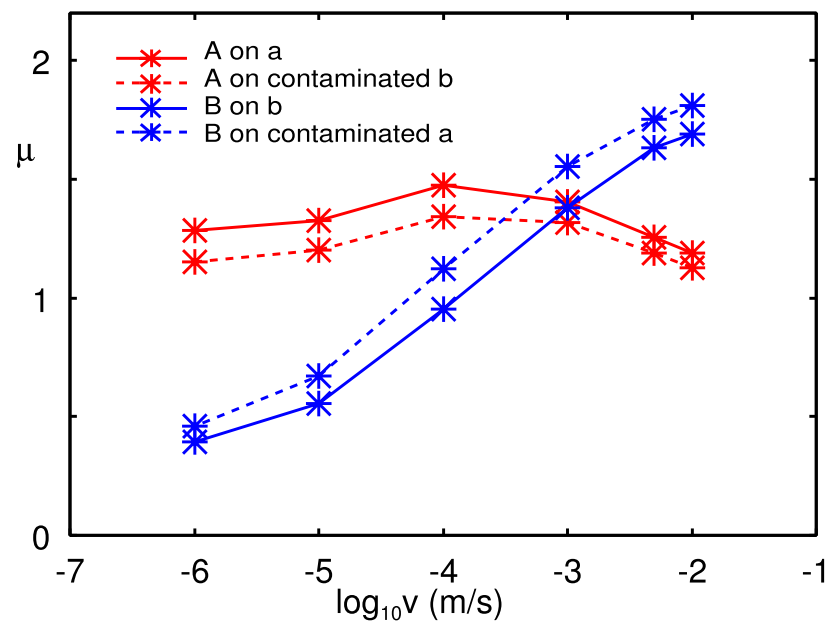

Fig. 4 The friction coefficient as a function of the logarithm of the sliding speed. The solid lines are for the compound A (red line) and B (blue line) sliding on two different concrete blocks ( $\mathbf{a}$ and $\mathbf{b}$, respectively) after run-in on the same concrete blocks (resulting in "contaminated" concrete surfaces). The red dashed line is for compound A sliding on the concrete block $\mathbf{b}$ after it was contaminated by the compound $\mathrm{B}$. The blue dashed line is for compound $\mathrm{B}$ sliding on the concrete block a after it was contaminated by the compound $\mathrm{A}$

of loose rubber particles the friction increases, or is nearly unchanged as observed below for another case.

In Sect. 4 we show that the friction for compound $A$ is mainly due to the viscoelastic deformations of the rubber by the road asperities. This contribution may be reduced more on the surface run-in by the compound $\mathrm{B}$, then on the surface run-in by compound A, since the former surface is covered by a much thicker transfer film which may effectively smooth the concrete surface at short length scale, and reduce the viscoelastic contribution to the friction.

\subsection{Compound C and D}

We have performed a similar set of experiments for compound $\mathrm{C}$ and $\mathrm{D}$ on the concrete surfaces $\mathbf{c}$ and $\mathbf{d}$. Figure 5 shows the friction coefficient during run-in at the sliding speed $v=1 \mathrm{~cm} / \mathrm{s}$ as a function of the number of sliding cycles for compound $\mathrm{C}$ (red) and D (blue) sliding on two different concrete blocks denoted $\mathbf{c}$ and $\mathbf{d}$, respectively.

Figure 6 shows picture of concrete blocks after run-in of (a) compound $\mathrm{C}$ on concrete block $\mathbf{c}$ and (b) compound D on concrete block $\mathbf{d}$. Note that just as for the racing compound A the wear occurs by formation of dry (non-sticky) rubber particles which are easily removed with a soft brush or with a vacuum cleaner.

The solid lines in Fig. 7 shows the friction coefficient as a function of the logarithm of the sliding speed for the compound $\mathrm{C}$ (red lines) and $\mathrm{D}$ (blue lines) sliding on the two

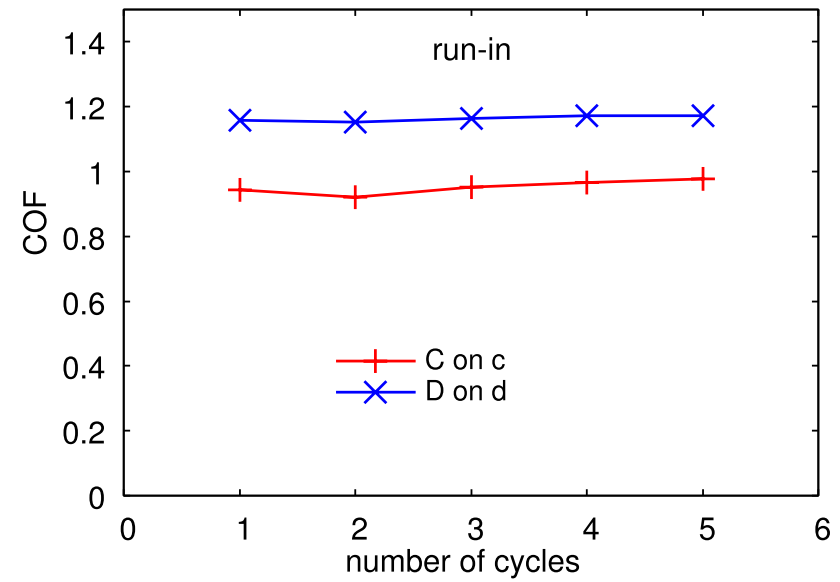

Fig. 5 The friction coefficient at the sliding speed $v=1 \mathrm{~cm} / \mathrm{s}$ as a function of the number of sliding cycles for compound $\mathrm{C}$ (red) and $\mathrm{D}$ (blue) sliding on two different concrete blocks denoted $\mathbf{c}$ and $\mathbf{d}$, respectively

different concrete blocks (c and $\mathbf{d}$, respectively) after run-in on the same concrete blocks (resulting in "contaminated" concrete surfaces). The red dashed line is for compound $\mathrm{C}$ sliding on the concrete block $\mathbf{d}$ after it was contaminated by the compound $\mathrm{D}$. The blue dashed line is for compound $\mathrm{D}$ sliding on the concrete block $\mathbf{c}$ after it was contaminated by the compound C. For compound D, there is nearly no difference in the friction when sliding on the two different concrete surfaces, but for compound $\mathrm{C}$ the friction is larger by $\sim 0.2$ on the surface contaminated by the compound D. Note that the friction coefficient depends relatively weakly on the sliding speed, as also found for the racing compound A.

Figure $6 \mathrm{c}, \mathrm{d}$ show pictures of concrete blocks $\mathbf{c}$ and $\mathbf{d}$ after performing all the experiments reported on above. In the measurements the rubber block start to move from the lower (darkest) contact region with the lowest speed $1 \mu \mathrm{m} / \mathrm{s}$ for $3 \mathrm{~cm}$, followed by the next lowest speed $10 \mu \mathrm{m} / \mathrm{s}$ for $3 \mathrm{~cm}$, and so on. Note that the wear decreases rapidly with increasing sliding speed. This is consistent with the rapid drop in the area of real contact with increasing sliding speed (see Fig. 11 below) and will be discussed further in Sect. 5 .

We also performed some sliding friction studies in water. After performing all the experiments discussed above we added water to the concrete surfaces $\mathbf{c}$ and $\mathbf{d}$ so the water level was about $\sim 3 \mathrm{~mm}$ above the highest asperities. Figure 8 shows the friction coefficient as a function of the logarithm of the sliding speed. The solid lines are for compound C (red) and D (blue) sliding on the dry concrete blocks $\mathbf{c}$ and d, respectively, after run-in on the same concrete blocks (the same as the solid lines in Fig. 7). The dashed lines are for compound C (red) and D (blue) sliding on the same two concrete surfaces in water. Note that in water there is a reduction in the friction coefficients for both compounds. This could be due either to a 


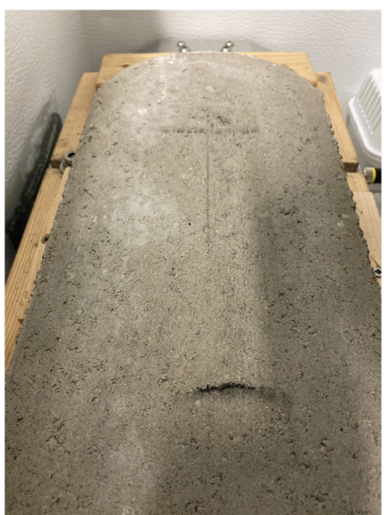

(a) run-in of $\mathrm{C}$ on $\mathrm{C}$ $[\mathrm{v}=1 \mathrm{~cm} / \mathrm{s}, 5$ cycles]

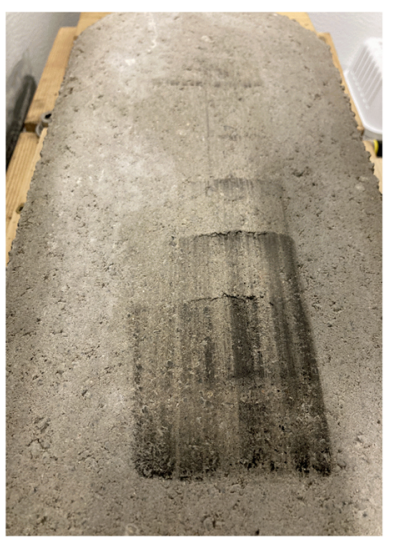

(c) run of $\mathrm{C}$ on $\mathrm{C}$ $[\mathrm{v}=1 \mu \mathrm{m} / \mathrm{s}, 10 \mu \mathrm{m} / \mathrm{s}, 100 \mu \mathrm{m} / \mathrm{s}, 1 \mathrm{~mm} / \mathrm{s}, 1 \mathrm{~cm} / \mathrm{s}]$

Fig. 6 Pictures of concrete blocks after run-in (at the velocity $v=1 \mathrm{~cm} / \mathrm{s}$ ) of (a) compound $\mathrm{C}$ on concrete block $\mathbf{c}$ and (b) compound D on concrete block $\mathbf{d}$. In (c) and (d) we show pictures of concrete blocks $\mathbf{c}$ and $\mathbf{d}$ after performing all the experiments reported on in this work. The sliding speed increases in steps of a factor of 10 from the bottom (most black area) to the top starting with $v=1 \mu \mathrm{m} / \mathrm{s}$ and ending with $1 \mathrm{~cm} / \mathrm{s}$. For each velocity the sliding distance $3 \mathrm{~cm}$

reduction in the adhesive contribution to the friction (but the theory predict rather small adhesive contribution even in the dry state), or due to a reduction in the viscoelastic contribution from sealed off water islands occurring at short length scale.

\section{Theory: Friction}

In this section, we present calculated results for the friction coefficient and the area of real contact as a function of the sliding speed. In the calculations, we use the measured viscoelastic modulus of compounds $\mathrm{A}-\mathrm{D}$, and the substrate is assumed rigid with the surface roughness power spectrum of the (clean) concrete surfaces used in the study in Ref. [1, 2].

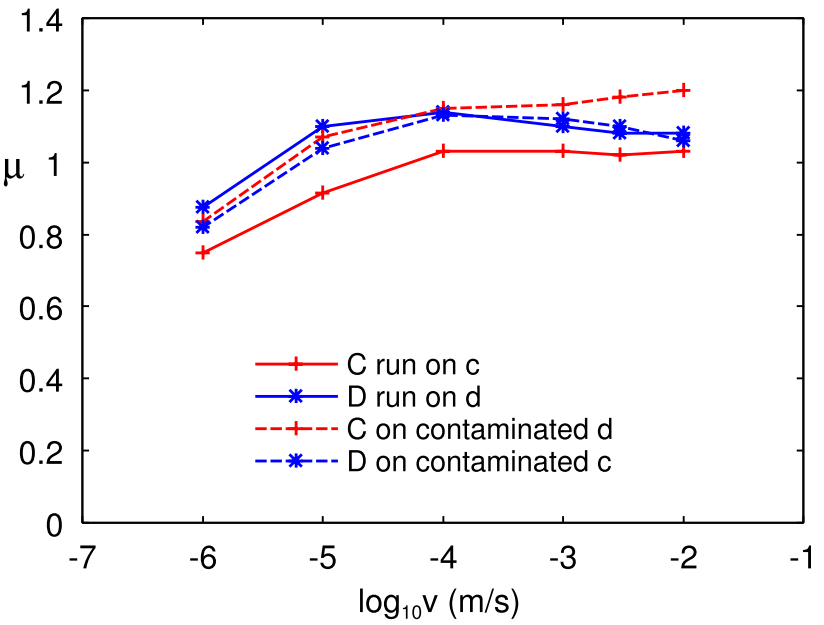

Fig. 7 The friction coefficient as a function of the logarithm of the sliding speed. The solid lines are for the compound $\mathrm{C}$ (red line) and $\mathrm{D}$ (blue line) sliding on two different concrete blocks (c and d, respectively) after run-in on the same concrete blocks (resulting in "contaminated" concrete surfaces). The red dashed line is for compound $\mathrm{C}$ sliding on the concrete block $\mathbf{d}$ after it was contaminated by the compound D. The blue dashed line is for compound D sliding on the concrete block $\mathbf{c}$ after it was contaminated by the compound $\mathrm{C}$

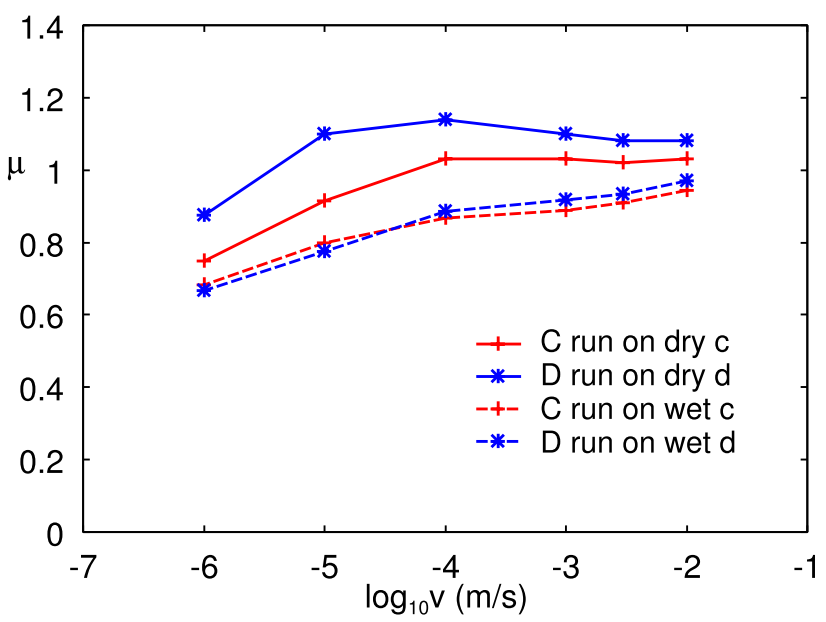

Fig. 8 The friction coefficient as a function of the logarithm of the sliding speed. The solid lines are for compound C (red line) and D (blue line) sliding on two dry concrete blocks (c and d, respectively) after run-in on the same concrete blocks. The dashed lines are for the compound C (red) and D (blue) sliding on the same two concrete blocks in water

The friction coefficient $\mu=\mu_{\mathrm{visc}}+\mu_{\mathrm{con}}$ is the sum of a viscoelastic contribution $\mu_{\mathrm{visc}}$, and a contribution from the area of real contact $\mu_{\text {con }}$, and depends on the sliding speed and the temperature as discussed elsewhere [3].

The contribution from the area of real contact may involve shearing a thin contamination film, or processes where 
polymer segments (or nanosized rubber patches) undergo cyclic stick-slip events [4-6], or where hard filler particles at the rubber surface scratches the substrate (plowing friction). The viscoelastic contribution to the friction results from the time-dependent deformations of the rubber by the substrate asperities, which occurs on many length scales [7].

We can write $\mu_{\text {con }}=\left(\tau_{\mathrm{f}} / p_{0}\right)\left(A / A_{0}\right)$, where the area of real contact $A$ depends on the contact pressure, temperature and the sliding speed. In the experiments (Sect. 3) the nominal contact pressure $p_{0} \approx 0.12 \mathrm{MPa}$ and the nominal contact area $A_{0} \approx 3 \times 7=21 \mathrm{~cm}^{2}$. The frictional shear stress $\tau_{\mathrm{f}}$ can originate from different processes which we now discuss.

The frictional shear stress depends on surface energies, contamination films and the location of slip (or shear) planes. One can distinguish between three limiting cases, namely (a) the surface has a thin, strongly bound, contamination film (usually water and polar molecules with a hydrocarbon part), or (b) a thicker contamination film occurs which could result from transfer of molecules from the rubber to the substrate surface, or (c) a weakly bound contamination film where slip occurs between the contamination film and the substrate surface.

If the contamination film is very thin (some nanometers), and the molecules bound strongly enough to the substrate surface (here concrete), slip will occur between the rubber and the contamination film. In this case the contribution from the area $A$ of real contact to the friction may involve interfacial processes where polymer segments, or nanosized patches of the rubber, attach, stretch, snap-off and re-attach to the substrate [4-6]. During the rapid snap-off the elastic energy in the stretched polymer chains are converted into heat. Theoretical [4-6] and experimental [3, 8] studies have shown that this results in a frictional shear stress which is well approximated by a Gaussian-like function of the logarithm of the sliding speed $v$, with a width of order several decades in velocity:

$\tau_{\mathrm{f}} \approx \tau_{0} \exp \left(-c\left[\log \frac{v}{v^{*}}\right]^{2}\right)$

where $v^{*}(T)$ depends on the temperature $T$, but which for $T=T_{0} \approx 20^{\circ} \mathrm{C}$ typically is of order $1 \mathrm{~cm} / \mathrm{s}$. We believe that in the present applications the shear stress $\tau_{\mathrm{f}}$ is likely due to the interaction between segments of rubber molecules on the rubber block with a rubber transfer film deposited on the substrate surface already at the leading edge of asperity contact regions. We believe that a shear plane forms at the interface between the transfer film and the rubber block and that most slip and (adhesive) energy dissipation occurs at this interface. Thus for dry contact the chemical nature of the substrate may not be important [9].

The temperature dependency
$v^{*}(T)=v^{*}\left(T_{0}\right) / a_{T}^{\prime}$,

where we have found, by analyzing experiments for different rubber compounds [3], that the shift factor $a_{T}^{\prime}$ is of the Arrhenius type

$\ln a_{T}^{\prime}=\frac{\epsilon}{k_{\mathrm{B}}}\left(\frac{1}{T}-\frac{1}{T_{0}}\right)$

with the activation energy $\epsilon \approx 1 \mathrm{eV}$ and $k_{\mathrm{B}}$ is the Boltzmann constant.

The parameters $\tau_{0}, v^{*}$ and $c$ in (1) cannot be predicted theoretically, but have to be determined by fitting to experimental friction results. In the calculations reported on below we have used $\mu_{\text {con }}=\left(\tau_{\mathrm{f}} / p_{0}\right)\left(A / A_{0}\right)$ with $\tau_{\mathrm{f}}$ given by (1) with $\tau_{0}=8 \mathrm{MPa}$ for compound $\mathrm{B}$ and for the racing compounds A,C,D $\tau_{0}=4 \mathrm{MPa}$, and with $c$ chosen so that the full-widthat-half-maximum of the Gaussian (1) is 4 velocity decades in all cases. At $T=20^{\circ}$ the Gaussian is centered at $v^{*}=5 \mathrm{~cm} / \mathrm{s}$ for compound B and $v^{*}=1 \mathrm{~mm} / \mathrm{s}$ for the compounds A,C,D. These values are very typical [3]. Note that the reference velocity $v^{*}$ in general decreases as the rubber glass transition temperature increases, and the values used here are consistent with this observation. To determine the parameters $\tau_{0}, c$ and the reference velocity $v^{*}$ more accurately it is necessary to measure the friction coefficient in a wider velocity range (or for more temperatures) then done here.

The viscoelastic contribution to the friction $\mu_{\text {visc }}$, and the relative contact area $A / A_{0}$, are calculated using the Persson rubber friction theory (see Ref. [1] for details).

If the contamination film is thicker it may behave as a (usually shear thinning) viscous fluid, and the shear stress acting on the rubber surface will be determined by the viscosity (and shear rate) of the fluid:

$\tau_{\mathrm{f}} \approx \frac{\eta_{0} \dot{\gamma}}{1+\left(\eta_{0} / B\right) \dot{\gamma}^{n}}$

where $B$ is a shear-thinning constant and $\dot{\gamma}=v / d$ the shear rate ( $d$ is the film thickness), and $\eta_{0}$ the low strain-rate viscosity. For polymer fluids the exponent $n$ is typically in the range $0.7-1[10,11]$, where the larger $n$ correspond to longer chain molecules.

In the present applications, we believe that a slip plane forms between the rubber and the contamination film. At this slip plane molecular stick-slip procceses occur and the frictional shear stress is given by (1).

For a smooth low-energy substrate surface, some of the slip may be localized at the interface between the substrate and the contamination film. However, the study in Ref. [12] indicates that even for very inert substrate the main slip may occur either within the contamination film or at the interface between the rubber and the contamination film. 


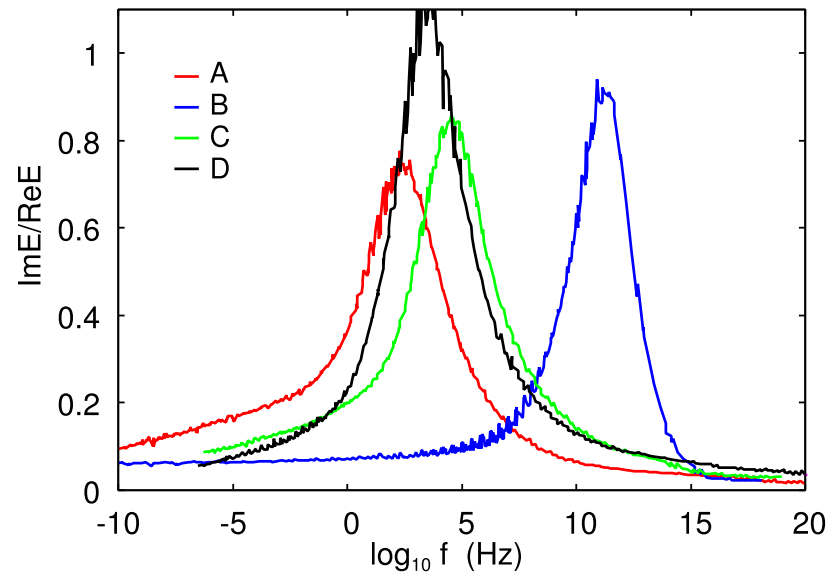

Fig. 9 The loss tangent $\tan \delta=\operatorname{Im} E / \operatorname{Re} E$ as a function of the logarithm of the frequency for compounds $\mathrm{A}, \mathrm{B}, \mathrm{C}$ and $\mathrm{D}$. At $T=20^{\circ} \mathrm{C}$ and for the strain 0.0004

To calculate the rubber friction, we need the viscoelastic modulus of the rubber and the surface roughness power spectrum of the concrete surface. The latter was obtained elsewhere (see, e.g., [1, 2]), and the former was obtained using a Dynamical Mechanical Analyse instrument as discussed in Appendix A.

Figure 9 shows the measured loss tangent $\tan \delta=\operatorname{Im} E / \operatorname{Re} E$ as a function of the logarithm of the frequency for compounds A,B,C and D obtained at $T=20^{\circ} \mathrm{C}$ and for the strain 0.0004 . Note that the racing compounds $\mathrm{A}, \mathrm{C}, \mathrm{D}$ have the maximum in $\tan \delta$ at much lower frequency then the bus/track compound $\mathrm{B}$, which reflect the fact that the glass transition of the compounds A,C,D are much higher than for the compound B (see Table 1).

\subsection{Compound A and B}

Figure 10 shows the calculated friction coefficient $\mu$ as a function of the logarithm of the velocity for compound $\mathrm{A}$ (red curves) and B (blue curves) at $T=20^{\circ} \mathrm{C}$. The dashed lines are the viscoelastic contribution $\mu_{\mathrm{visc}}$ and the solid lines the sum $\mu=\mu_{\mathrm{visc}}+\mu_{\mathrm{con}}$ of the viscoelastic contribution and the (adhesive) contribution from the area of real contact. The total friction coefficient is in good semi-quantitative agreement with the measured data (compare Fig. 4 and 10). Note the small contribution to the friction from the area of real contact for the racing compound $\mathrm{A}$. This is due to the small area of real contact in the relevant velocity (or frequency) region resulting from the high glass transition temperature of compound A. This is illustrated in Fig. 11 which shows the relative contact area $A / A_{0}$ as a function of the logarithm of the velocity for compound A (red curve) and compound B (blue curve) at $T=20^{\circ} \mathrm{C}$.

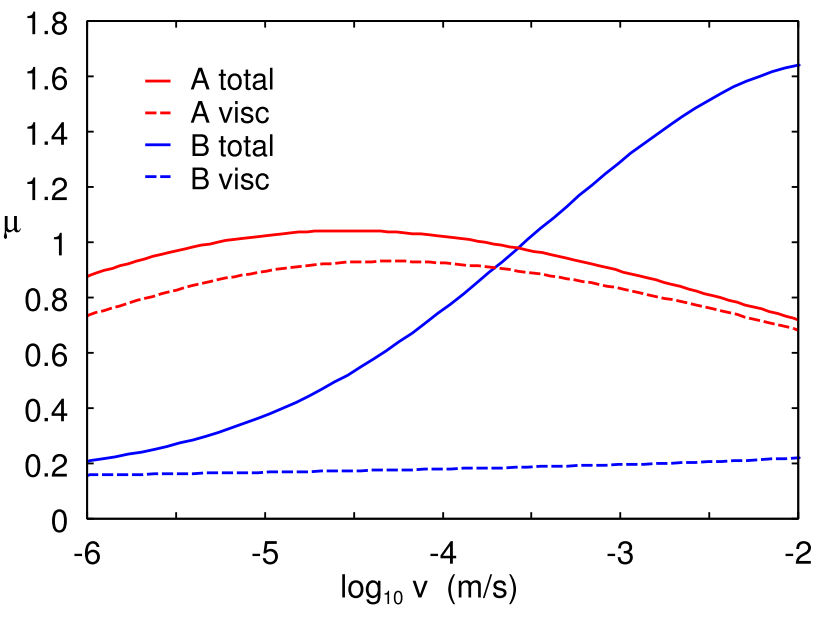

Fig. 10 The friction coefficient $\mu$ as a function of the logarithm of the velocity for compound A (red curves) and B (blue curves) at $T=20^{\circ} \mathrm{C}$. The dashed lines are the viscoelastic contribution $\mu_{\text {visc }}$ and the solid curves the sum $\mu=\mu_{\text {visc }}+\mu_{\text {con }}$ of the viscoelastic contribution and the (adhesive) contribution from the area of real contact

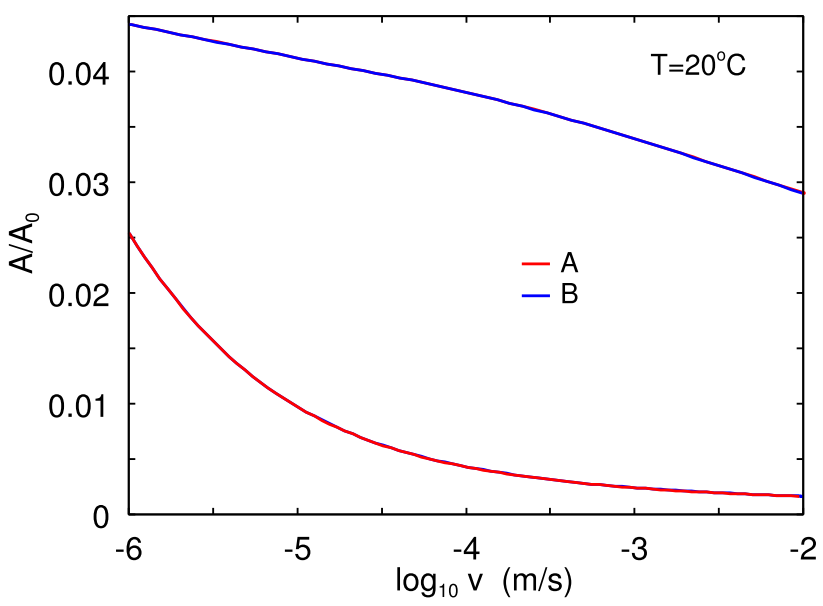

Fig. 11 The relative contact area $A / A_{0}$ as a function of the logarithm of the velocity for compound A (red curve) and compound B (blue curve) at $T=20^{\circ} \mathrm{C}$

The friction results for compounds A and B are consistent with the wear pattern observed in Sect. 3. Thus the small area of real contact predicted by the theory for compound A for $T=20^{\circ} \mathrm{C}$ and the sliding speed $v=1 \mathrm{~cm} / \mathrm{s}$ suggest that adhesive wear, which would result in the formation of a smear film on the concrete surface, is small for this compound during run-in, which is consistent with Fig. 3 which shows mainly wear by the formation of "dry" wear particles. However, for compound B, the friction is mainly due to adhesive interactions and the wear occurs via the formation of a smear film.

In F1-racing the tire surface temperature is typically $80^{\circ}-110^{\circ} \mathrm{C}$ and in Fig. 12 we show the friction coefficient 


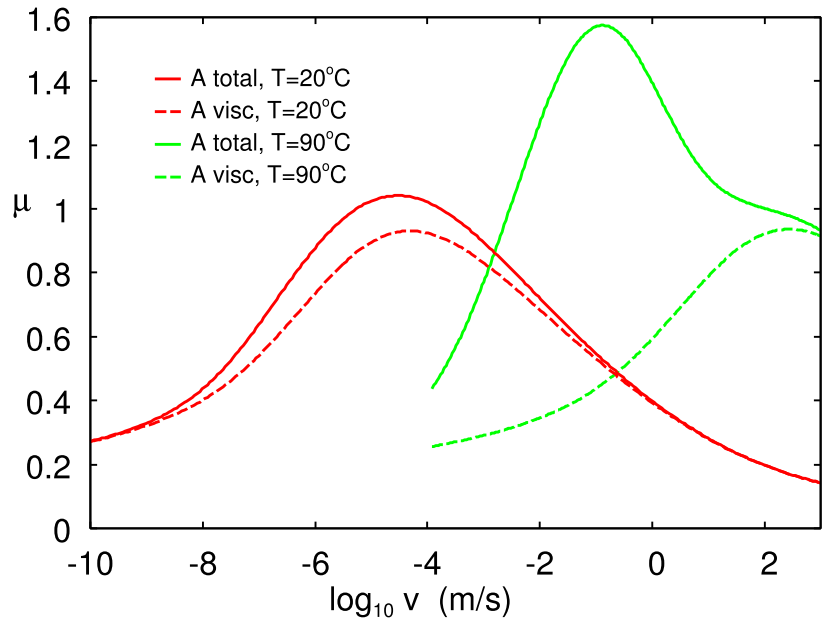

Fig. 12 The friction coefficient $\mu$ as a function of the logarithm of the velocity for compound A at $T=20^{\circ} \mathrm{C}$ (red curves) and $T=90^{\circ} \mathrm{C}$ (green curves). The dashed lines are the viscoelastic contribution $\mu_{\text {visc }}$ and the solid curves the sum $\mu=\mu_{\mathrm{visc}}+\mu_{\text {con }}$ of the viscoelastic contribution and the (adhesive) contribution from the area of real contact

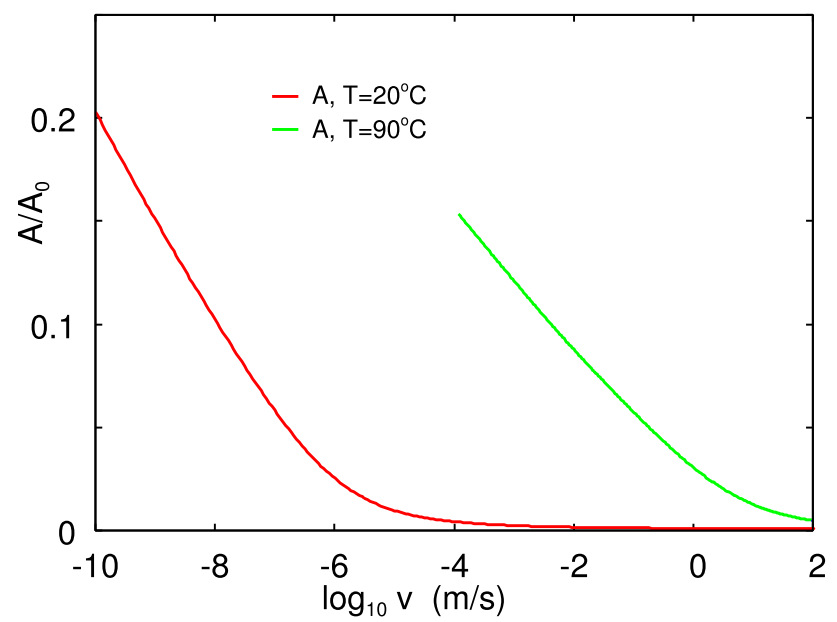

Fig. 13 The relative contact area $A / A_{0}$ as a function of the logarithm of the velocity for compound A at $T=20^{\circ} \mathrm{C}$ (red curve) and $T=90^{\circ} \mathrm{C}$ (green curve)

$\mu$ as a function of the logarithm of the velocity for compound A at $T=20^{\circ} \mathrm{C}$ (red curves) and $T=90^{\circ} \mathrm{C}$ (green curves). The dashed lines are the viscoelastic contribution $\mu_{\mathrm{visc}}$ and the solid curves the sum $\mu=\mu_{\mathrm{visc}}+\mu_{\mathrm{con}}$ of the viscoelastic contribution and the (adhesive) contribution from the area of real contact. Note that at $T=90^{\circ} \mathrm{C}$ the adhesive contribution to the friction is very important. This is due to an increase in the contact area resulting from the softening of the rubber with increasing temperature. This is illustrated in Fig. 13 which shows the relative contact area $A / A_{0}$ as a function of the logarithm of the velocity for compound A at $T=20^{\circ} \mathrm{C}$ (red curve) and $T=90^{\circ} \mathrm{C}$ (green curve).
Note that at $T=90^{\circ} \mathrm{C}$ the sliding friction coefficient for the racing compound $\mathrm{A}$ is of order 1.4-1.6 for sliding speeds of order $\sim 0.1-1 \mathrm{~m} / \mathrm{s}$. This appears to be consistent with practical experience for racing tires where the maximum in the $\mu$ - slip curve (which typically occurs for (average) slip velocities of order $1 \mathrm{~m} / \mathrm{s}$ ) typically is of order 1.4-1.5.

\subsection{Compound C and D}

In Fig. 14, we show the friction coefficient $\mu$ as a function of the logarithm of the velocity for compound $\mathrm{C}$ (red curves) and D (blue curves) at $T=20^{\circ} \mathrm{C}$. The dashed lines are the viscoelastic contribution $\mu_{\mathrm{visc}}$ and the solid curves the sum $\mu=\mu_{\text {visc }}+\mu_{\text {con }}$ of the viscoelastic contribution and the (adhesive) contribution from the area of real contact. Note that for the speed $v=1 \mathrm{~cm} / \mathrm{s}$ for both compounds the friction coefficient is dominated by the viscoelastic contribution while for the lowest speed the adhesive contribution from the area of real contact is very important. This is, in part, due to the strong increase in the area of real contact with decreasing sliding speed. This is illustrated in Fig. 15 which shows the relative contact area $A / A_{0}$ as a function of the logarithm of the velocity for both compounds. The velocity dependency of $\mu_{\mathrm{visc}}$ and $\mu_{\text {con }}$ and of the contact area will now be used to discuss the dependency of the nature of the wear on the sliding speed (Fig. 6).

\section{Rubber Wear}

Figure 3 and 6 show that only compound B gives rise to a smear film on the concerete surface during run-in. The other compounds A,C,D wear by formation of a dry powder

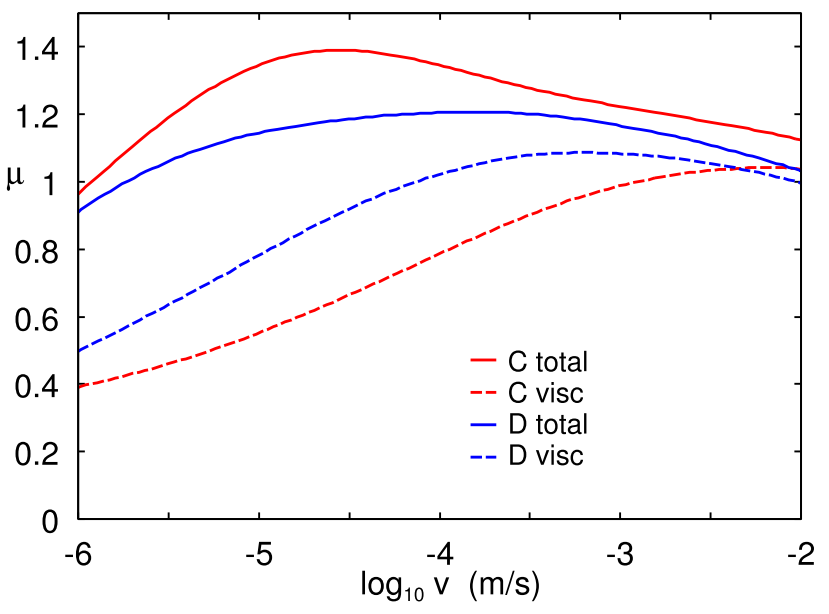

Fig. 14 The friction coefficient $\mu$ as a function of the logarithm of the velocity for compound $\mathrm{C}$ (red curves) and $\mathrm{D}$ (blue curves) at $T=20^{\circ} \mathrm{C}$. The dashed lines are the viscoelastic contribution $\mu_{\mathrm{visc}}$ and the solid curves the sum $\mu=\mu_{\text {visc }}+\mu_{\text {con }}$ of the viscoelastic contribution and the (adhesive) contribution from the area of real contact 


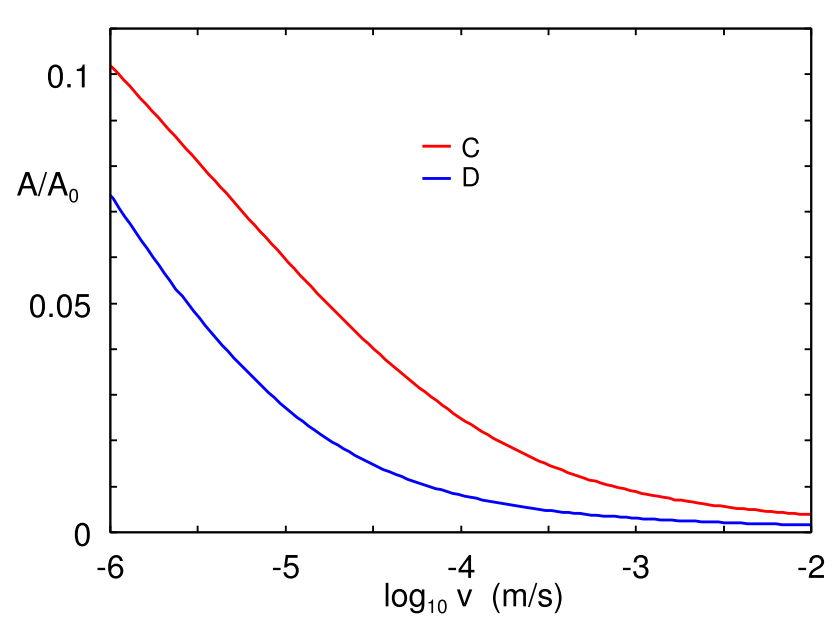

Fig. 15 The relative contact area $A / A_{0}$ as a function of the logarithm of the velocity for compound $\mathrm{C}$ (red curve) and compound $\mathrm{D}$ (blue curve) at $T=20^{\circ} \mathrm{C}$

of rubber particles. Now, when sliding compound A on the surface covered by a smear film of compound B (case A on contaminated $\mathbf{b}$ in Fig. 4) the friction is lower that when compound $\mathrm{A}$ is sliding on the surface contaminated by compound A (A on a). Thus, the smear film created by compound $\mathrm{B}$ reduces the friction while the loose particles created by the compound A do not. This is consistent with the results for compound $\mathrm{C}$ and $\mathrm{D}$ where sliding on the contaminated surfaces (with loose particles) results in higher or similar friction.

In the context of rubber wear of tires one speak about two limiting wear modes, namely wear resulting in a "dry powder" of rubber particles, or in a sticky smear film. But this does not necessarily correspond to rubber wear products with different chemical composition, but rather may just reflect the size of the wear particles. Thus it is known that adhesion is killed by the long wavelength roughness [13, 14]. The longest wavelength roughness of a wear particle is determined by the linear size of the particle. Thus small rubber wear particles will always be sticky and adhere to surfaces (e.g., the substrate surface, or any other surface), effectively forming a smear film. If the particles are big enough, and have large enough surface roughness (as expected for wear particles), they will not adhere to themself or to the substrate surface forming a "dry powder". This is also what is observed experimentally.

One expect different wear processes associated with the viscoelastic contribution and the adhesive contribution to the rubber friction. Thus the adhesive contribution may generate large stresses in the area of real contact, but the area of real contact may consist of (very many) very small contact regions. The shear stress acting in these contact region is unlikely to generate wear particles with lateral dimension larger than the width of the contact regions, unless they are very closely spaced [15]. The viscoelastic contribution to the friction act on many length scales since asperity size spreads many length scales. In general, the stress field from an asperity (apparent) contact regions can only drive cracks into the rubber a distance determined by the linear size of the (apparent) asperity contact region. But since a wide distribution of asperities occur we expect a wide distribution of wear particle sizes to result from the viscoelastic contribution to the friction as discussed in detail in Reference [16, 17].

We note also that even if the frictional shear stress is relative small, detachment of rubber particles can occur due to the mechanochemical nature of the bond breaking process. That is, the barrier for bond breaking is lowered by the simultaneous formation of new bonds with, e.g., oxygen atoms. Thus, it has been observed that the degradation of filled NR and SBR to a sticky material occurs only in the presence of oxygen, but not in a nitrogen atmosphere [18]. Bond breaking is also affected by thermal fluctuations which can supply part of the energy needed to overcome the barrier towards breaking a bond. However, due to the low sliding speed negligible frictional heating is expected in our study so thermal effects are likely not to be enhanced by the sliding motion in our case.

That oxygen is involved in the bond breaking process is known from experiments since the rubber wear particles have a higher concentration of bound oxygen than the original rubber. This is particularly true when the rubber wear occurs by the formation of a smear film. Thus if small wear particles or "smear" is formed, as may be associated with the adhesive contribution to the friction, the wear products are likely to have a higher concentration of oxygen atoms than big wear particles due to the higher surface to volume ratio.

Figure $6 c, d$ show pictures of concrete blocks $\mathbf{c}$ and $\mathbf{d}$ after performing all the experiments reported on above. In last measurements the rubber block start to move from the lower (darkest) contact region with the lowest speed $1 \mu \mathrm{m} / \mathrm{s}$ for $3 \mathrm{~cm}$, followed by the next lowest speed $10 \mu \mathrm{m} / \mathrm{s}$ for $3 \mathrm{~cm}$, and so on. Note that the wear decreases rapidly with increasing sliding speed. This is consistent with the rapid drop in the area of real contact with increasing sliding speed (see Fig. 15). At the highest speed $1 \mathrm{~cm} / \mathrm{s}$ the wear is very small and consist mainly of "dry powder" which accumulates at the turn-around regions of the sliding track as in Fig. 6a, b. At the lowest sliding speed the wear occurs by the formation of a smear film, i.e., a different wear process. This is correlated with the fact that the viscoelastic contribution to the friction dominate for the largest sliding, while at the lowest speed the adhesive contribution from the area of real contact becomes very important (see Fig. 15). Note also that the contact time is 10000 times longer for the lowest sliding speed compared to the highest sliding speed, which may increase the wear as thermal and mechanochemical processes become more important with increasing contact time. 


\section{Summary and Conclusion}

We have shown that there is a slight decrease in the rubber friction coefficient with the number of run-in cycles for compounds $\mathrm{A}$ and $\mathrm{B}$, but a negligible change for compounds $\mathrm{C}$ and $\mathrm{D}$ (but these compounds was run-in for a shorter time). When a rubber block is sliding on a concrete surface, which is contaminated by another rubber compound, the friction force may be either bigger or smaller than if sliding occurs on a surface contaminated by the same type of rubber compound. In an earlier study, [9] for two very different rubber compounds similar results was obtained as found above. The present study indicates that the nature of the contamination film may be important when predicting if the friction for a second rubber compound will increase or decrease by the contamination film. Hence a smear film results in a reduction in the friction while when the wear occurs by formation of loose rubber particles the friction is nearly unchanged or increased.

The change in the friction coefficient between the different systems is relative small, typically about $\sim 10 \%$. This change may of course be crucial in some applications like in F1-racing. We note, however, that our experimental study was for the temperature $T=20^{\circ} \mathrm{C}$, and tire temperatures are often higher (but not necessarily the road temperature), and in F1-racing much higher, typically $T=80-110^{\circ} \mathrm{C}$. In addition, racing tire tread compounds have additives (e.g., resin) which become sticky only at high temperatures (say above $80^{\circ} \mathrm{C}$ ). Experimental studies at higher temperatures for racing compounds are necessary to determine if this will change the conclusions obtained above. F1 racing typically occur on asphalt road surfaces, but we do expect similar wear and frictional properties on such surfaces as for the concrete surface used here.

We have present theory results which illustrate the relative importance of the viscoelastic and adhesive contribution to the sliding friction. For the racing compounds A,C,D we found that at $T=20^{\circ} \mathrm{C}$ the viscoelastic contribution dominate the friction for $1 \mathrm{~cm} / \mathrm{s}$ while for the bus/truck compound B the adhesive contribution dominates, and we attribute the different wear mode observed to this fact. Thus, for the racing compound the wear consist of "dry" (non-adhesive) rubber particles which are easily removed with a soft brush or a vacuum cleaner. For the compound B, we instead found a sticky smear layer which cannot be removed from the concrete surface by mechanical means. We attributed this difference to the size of the wear particles which depends on what process (adhesive or visdcoelastic) dominate the friction force.

\section{Appendix A}

For rubber friction it is necessary to have information about the complex elastic modulus over a rather large frequency range, as well as at different strain values including very large strain of order $100 \%$. A standard way of measuring the viscoelastic modulus is to oscillatory deform the rubber sample with a constant strain or stress amplitude. This is done at different frequencies and then repeated at different temperatures. The results measured at different temperatures can be time-temperature shifted to a master curve at a chosen reference temperature covering a broad range of frequencies.

Here we present results for the viscoelastic properties for the rubber compounds A-D used above. For our measurements, we use a Q800 Dynamic Mechanical Analysis (DMA) instrument produced by TA Instruments. The machine is run in tension mode, meaning that a strip or a fiber of rubber clamped on both sides, is elongated in an oscillatory manner. The complex viscoelastic modulus is first measured in constant strain mode with a strain amplitude of $0.04 \%$ strain and at different frequencies starting from $28 \mathrm{~Hz}$ and changed in steps until $0.25 \mathrm{~Hz}$ is reached (10 frequency points: 28.0, 25.0, 14.0, 7.9, 4.4, 2.5, 1.4, $0.79,0.44$, and $0.25 \mathrm{~Hz}$ ). The rather small strain amplitude is chosen to avoid strain softening effects. It is not clear how these nonlinear effects would affect the results at different temperatures, and one therefore usually measure the low strain master curve in the linear response region. We have found that a strain amplitude of $0.04 \%$ is reasonably good for typical tread rubber compounds. Measuring the rubber sample in tension mode also requires to prestrain the rubber with a static strain that has to be larger then the dynamic strain during oscillation. The prestrain in the experiments has been set to $0.06 \%$ to avoid compressing the rubber during the DMA measurement.

The experiment usually starts at $-75^{\circ} \mathrm{C}$ and after measuring the modulus at all frequencies mentioned above, the temperature is increased in steps by $5^{\circ} \mathrm{C}$, until the transition region is reached. In the transition region, we increase the temperature step by $2.5^{\circ} \mathrm{C}$ until the onset of rubbery region and after that we switch back to increasing temperatures in steps of $5^{\circ} \mathrm{C}$ until the end temperature of $120^{\circ} \mathrm{C}$ or $150^{\circ} \mathrm{C}$ is reached. Note that it may be necessary to choose smaller temperature steps when reaching the glass transition temperature $T_{\mathrm{g}}$ where the viscoelastic response of the rubber material changes strongly with frequency (and temperature). This makes sure that the curves measured at different temperatures overlap with each other, which is necessary for the shift procedure. The results are then shifted to form a smooth $\operatorname{Re} E$ master curve.

Figure 16 shows the (a) real $\operatorname{Re} E$ and (b) the imaginary $\operatorname{Im} E$ part of the viscoelastic modulus $E$ as a function of the 

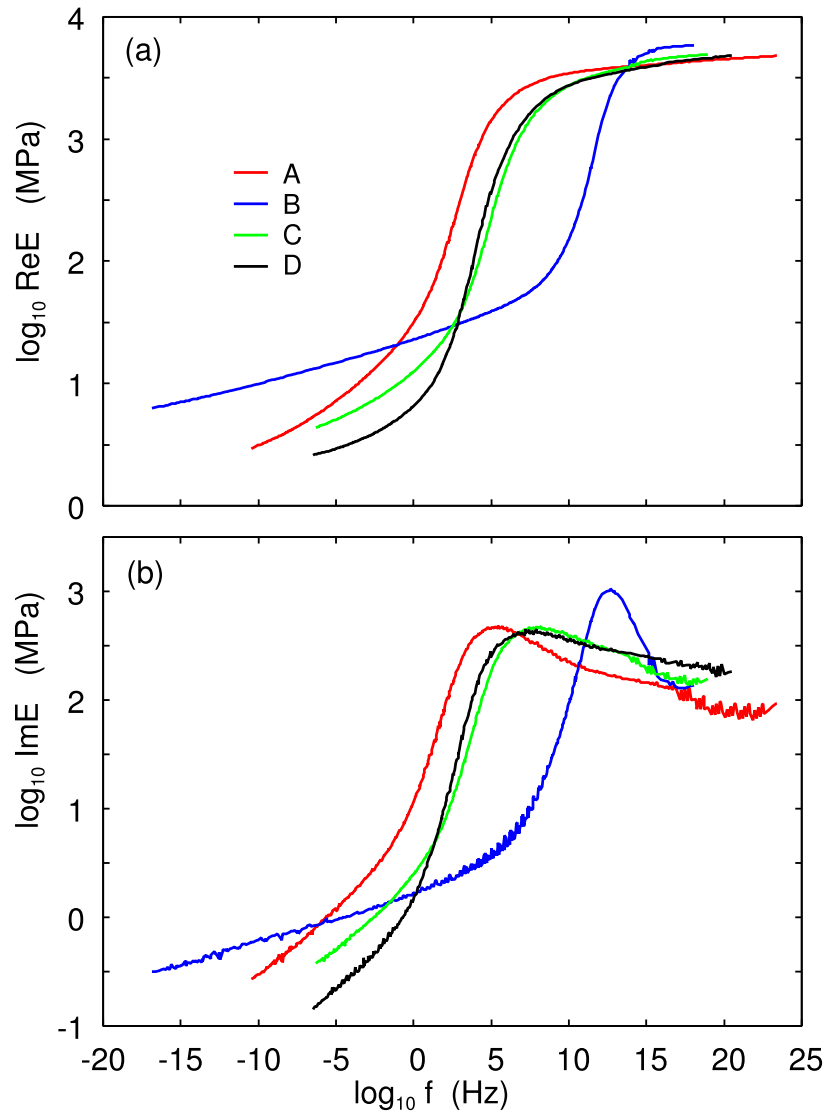

Fig. 16 The (a) real $\operatorname{Re} E$ and (b) the imaginary $\operatorname{Im} E$ part of the viscoelastic modulus as a function of the frequency for compounds $\mathrm{A}, \mathrm{B}$, $\mathrm{C}$ and D (log-log scale). At $T=20^{\circ} \mathrm{C}$ and for the strain 0.0004

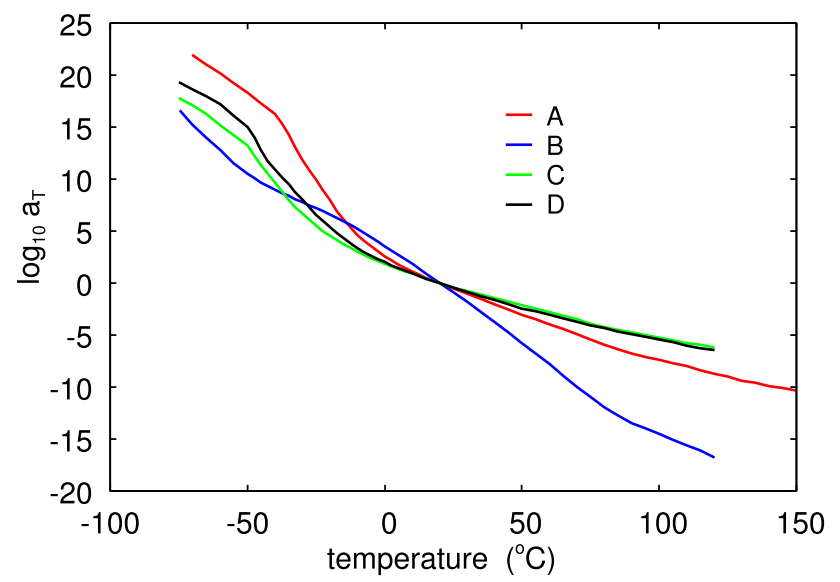

Fig. 17 The logarithm of the horizontal shift factor $a_{T}$ as a function of temperature for compounds A, B, C and D (log-log scale). The reference temperature $T=20^{\circ} \mathrm{C}$

frequency for compounds A, B, C and D (log-log scale). The horizontal shift factor $a_{T}$ needed to produce these mastercurves is shown in Fig. 17 as a function of temperature.

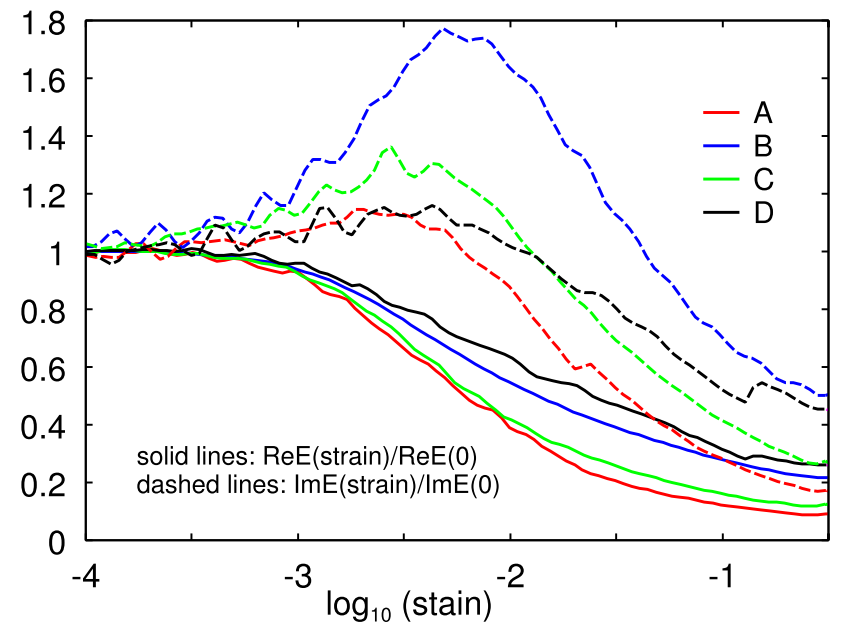

Fig. 18 The ratio $\operatorname{Re} E(\epsilon) / \operatorname{Re} E(0)$ (solid lines) and $\operatorname{Im} E(\epsilon) / \operatorname{Im} E(0)$ (dashed lines) as a function of the logarithm of the strain $\epsilon$ for compounds $\mathrm{A}, \mathrm{B}, \mathrm{C}$ and $\mathrm{D}$

We have also studied the large strain dependency of the effective viscoelastic modulus. The measurements were performed at the frequency $f=1 \mathrm{~Hz}$ for several different temperatures. Figure 18 shows the ratio $\operatorname{Re} E(\epsilon) / \operatorname{Re} E(0)$ (solid lines) and $\operatorname{Im} E(\epsilon) / \operatorname{Im} E(0)$ (dashed lines) as a function of the logarithm of the strain $\epsilon$ for compounds A, B, C and D. The results are averages of the strain curve obtained for the temperatures $T=-10,0,20,60$ and $100^{\circ} \mathrm{C}$.

Funding Open Access funding enabled and organized by Projekt DEAL.

Open Access This article is licensed under a Creative Commons Attribution 4.0 International License, which permits use, sharing, adaptation, distribution and reproduction in any medium or format, as long as you give appropriate credit to the original author(s) and the source, provide a link to the Creative Commons licence, and indicate if changes were made. The images or other third party material in this article are included in the article's Creative Commons licence, unless indicated otherwise in a credit line to the material. If material is not included in the article's Creative Commons licence and your intended use is not permitted by statutory regulation or exceeds the permitted use, you will need to obtain permission directly from the copyright holder. To view a copy of this licence, visit http://creativecommons.org/licenses/by/4.0/.

\section{References}

1. Lorenz, B., Oh, Y.R., Nam, S.K., Jeon, S.H., Persson, B.N.J.: Rubber friction on road surfaces: experiment and theory for low sliding speeds. J. Chem. Phys. 142, 194701 (2015)

2. Tolpekina, T.V., Persson, B.N.J.: Adhesion and friction for three tire tread compounds. Lubricants 7, 20 (2019) 
3. Tiwari, A., Miyashita, N., Espallargas, N., Persson, B.N.J.: Rubber friction: the contribution from the area of real contact. J. Chem. Phys. 148, 224701 (2018)

4. Schallamach, A.: A theory of dynamic rubber friction. Wear $\mathbf{6}$, 375 (1963)

5. Cherniak, Y.B., Leonov, A.I.: On the theory of the adhesive friction of elastomers. Wear 108, 105 (1986)

6. Persson, B.N.J., Volokitin, A.I.: Rubber friction on smooth surfaces. Eur. Phys. J. E 21, 69 (2006)

7. Persson, B.N.J.: Theory of rubber friction and contact mechanics. J. Chem. Phys. 115, 3840 (2001)

8. Tiwari, A., Dorogin, L., Tahir, M., Stöckelhuber, K.W., Heinrich, G., Espallargas, N., Persson, B.N.J.: Rubber contact mechanics: adhesion, friction and leakage of seals. Soft Matter 13, 9103 (2017)

9. Tiwari, A., Tolpekina, T., van Benthem, H., Gunnewiek, M.K., Persson, B.N.J.: Rubber adhesion and friction: role of surface energy and contamination films. Front. Mech. Eng. (2020). https ://doi.org/10.3389/fmech.2020.620233

10. Yamada, S.: General shear-thinning dynamics of confined fluids. Tribol. Lett. 13, 167 (2002)

11. Sivebaek, I.M., Samoilov, V.N., Persson, B.N.J.: Effective viscosity of confined hydrocarbons. Phys. Rev. Lett. 108, 036102 (2012)

12. Rotella, C., Persson, B.N.J., Scaraggi, M., Mangiagalli, P.: Lubricated sliding friction: role of interfacial fluid slip and surface roughness. Eur. Phys. J. E 43, 9 (2020)

\section{Authors and Affiliations}

\author{
A. Tiwari \\ avicm926@gmail.com \\ $\triangle$ B. N. J. Persson \\ b.persson@fz-juelich.de \\ 1 PGI-1, Jülich, Germany
}

A. Tiwari ${ }^{1,3}$ - N. Miyashita ${ }^{2}$ B. N. J. Persson ${ }^{1,3}$
13. Tiwari, A., Wang, J., Persson, B.N.J.: Adhesion paradox: why adhesion is usually not observed for macroscopic solids. Phys. Rev. E 102, 042803 (2020)

14. Persson, B.N.J., Sivebk, I.M., Samoilov, V.N., Zhao, K., Volokitin, A.I., Zhang, Z.: The origin of Amontons friction law. J. Phys. 20(39), 395006 (2008)

15. Phan-Ba, S., Brink, T., Molinari, J.F.: Adhesive wear and interaction of tangentially loaded micro-contacts. Int. J. Solids Struct. 188, 261 (2020)

16. Persson, B.N.J.: Theory of powdery rubber wear. J. Phys. Condensed Matter 21, 485001 (2009)

17. Rodriguez, N., Mangiagalli, P., Persson, B.N.J.: Viscoelastic crack propagation: review of theories and applications. Adv. Polym. Sci. 1, 15 (2020). https://doi.org/10.1007/12_2020_76

18. Schallamach, A.: Abrasion, fatigue and smearing of rubber. J. Appl. Polym. Sci. 12, 281 (1968)

Publisher's Note Springer Nature remains neutral with regard to jurisdictional claims in published maps and institutional affiliations

2 The Yokohama Rubber Company, 2-1 Oiwake, Hiratsuka, Kanagawa 254-8601, Japan

3 MultiscaleConsulting, Wolfshovener Str, 252428 Jülich, Germany 